\title{
Evaluation of proliferation activity of oral lichen planus and oral lichenoid reactions using quantitative and qualitative analysis of AgNORs (Argyrophilic Nucleolar Organizer Regions)
}

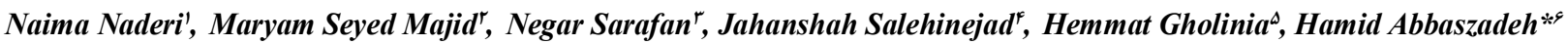

Received 22 Dec 2016, Accepted for publication 19 Feb, 2017

\begin{abstract}
Bachground \& Aims: Oral lichenoid reactions (OLR) and oral lichen planus (OLP) are clinically and histopathologically similar but their therapeutic approach are different. Due to the presence of dysplasia in oral lichen planus which effects prognosis, the aim of study was the evaluation of proliferation activity of OLPs and OLRs and their subtypes. AgNOR technique that is inexpensive and easy to perform was used to differentiate OLPs and OLRs and their subtypes.
\end{abstract}

Materials \& Methods: in this cross sectional, retrospective study, samples consisted of 45 OLPs, 30 OLRs 15 normal oral mucosa (N) from healthy persons (as control group). The samples were stained by AgNOR technique. AgNOR dots were counted on 100 cells from basal and para basal layer. The mean AgNOR dost per nucleus were calculated. Proliferation index (number of cells with AgNOR dots $\geq 5$ ) was also determined. Variation in size of AgNOR dots was categorized into 2 groups.

Result: There were significant differences between OLP(and its subtypes) and N and also between OLR(and erosive type) and N .

Such a difference was also between reticular OLP and reticular OLR.with regard to AgNOR count.

Conclusion: Since reticular OLP had significantly higher AgNOR count than reticular OLR in our study, we can suggest the use of AgNOR technique for differentiation of reticular type of OLP from OLR on challenging histopathologic cases.

Key words: lichen pelanus, cell proliferation, nucleolar organizer region associated protein.

Address: Velayat Sq, Noshirvani Blvd, Faculty of Dentistry, Babol University of Medical Sciences, Babol, Iran

Tel: (+98) 1132197667

Email: hamidabbaszade@yahoo.com

\section{Introduction}

Lichen planus is a chronic inflammatory disease mucocutaneous which was introduced for the first time by Mr. Erasmus Wilson in 1869(1). Its etiology in most cases are idiopathic and some factors such as degeneration of the basal layer by cell-mediated immunity are the most important cause of oral lichen planus (1).

Oral mucosa and skin may be show clinical and microscopic changes similar to those in Lichen Planus after taking some anti-rheumatoid drugs including nonsteroidal anti-inflammatory drugs called lichenoid

\footnotetext{
${ }^{1}$ Dental Student, student Research Committee, Babol University of Medical Sciences, Babol, Iran

${ }^{2}$ Associate professor of Dental Materials Research Center, University of Medical Sciences, Babol, Iran

${ }^{3}$ Assistant Professor, Department of Oral Medicine, Dental School, University of Medical Sciences, Urmia, Iran

${ }^{4}$ Associate Professor, Department of Oral and Maxillofacial Pathology, Dental School, Mashhad University of Medical Sciences, Mashhad, Iran

${ }^{5}$ MSc in Statistics, Department of social Medicneand Health, Babol University of Medical Sciences, Babol, Iran

${ }^{6}$ Assistant Professor, cellular and molecular biology research center, Department of Oral and Maxillofacial Pathology, Dental School,Babol

University of Medical Sciences, Babol, Iran (Corresponding Author)
} 
reactions (1). Among factors which may cause local or systemic etiologic cause, lichenoid reactions can be noted as restorative materials, metal and resin (2). Some sources are classified as lichen planus part of lichenoid reactions (3).

Multiple forms of systemic and topical antiinflammatory and immunosuppressive treatments for lichen planus have been considered. While the best therapeutic strategy to stop the reaction of Lichenoid is removing the local drive of its creator (1).

On the other hand the possibility oral lichen planus malignancy is discussed (4-5-6). Dysplasia in $11 \%$ to $25 \%$ has been reported (7) and malignant transformation in the $0 \%$ to $5 / 12 \%$ has been observed in patients with oral lichen planus (8-9). One of the noteworthy challenges about oral lichen planus is the diagnosis of presence of dysplasia in tissue sections derived from it. In some cases, it is difficult to express the presence of dysplasia in the histopathologic specimens. Considering the influence of pathologic report of dysplasia in oral lichen planus lesions on prognosis and drug treatment, laser therapy and more detailed follow-up surgery, finding cheap and easy technique to confirm the presence or absence of dysplasia in lichen planus patients is vital.

Increased proliferative capacity is one of the first indicators of malignant transformation as a key factor for cancer formation. Among numerous techniques used to make such an assessment, NORs qualitative and quantitative analysis, through histochemical AgNOR staining, particular attention are needed for the sake of simplicity, low cost and high reliability (10).

AgNOR techniques mark proteins associated with nucleolar organizer regions (NORs). NORs are loops of DNA that is transcribed ribosomal RNA. Non-histone proteins such as asidik and argyrophilic are associated with NOR. NORs can be shown by staining associate proteins of tissue sections by colloidal silver and these colored reagent products with silver are known as argyrophilic nucleolar organiser regions (AgNORs) (1011). The higher number of NORs, the lower cell cycle and speeder proliferation of cell (10).

Such a relationship makes NOR as a valuable indicator in the analysis of higher activity of cell proliferation and a diagnostic tool (10). However, some studies have shown that morphological characteristics of AgNOR contain useful information (12).

In the current research, with regard to the importance of recognizing the difference between lesions of oral lichen planus and lichenoid reactions, we tried to assess the proliferative activity of oral lichen planus and oral lichenoid reaction by an inexpensive and easy technique called AgNORs analysis.

\section{Materials and Methods}

In this retrospective cross-sectional study 45 cases of oral lichen planus (Including 15 cases of reticular, 15 cases of Erosive and 15 cases of oral lichen planus with dysplasia), 30 cases of oral lichenoid reactions (including 15 cases of reticular and 15 cases of erosive type) among textured blocks available at the archives of department of Oral \& Maxillofacial Pathology of Babol and Mashhad University of Medical Sciences since 2007 to 2015 were selected. 15 cases of normal mucosa were also considered for the control group. 4 micron sections were prepared from blocks and microscopic slides were investigated and confirmed by two independent pathologists. Based on histopathologic findings, the type of lichen planus and oral lichenoid reactions (reticular and erosive forms) according to the Neville reference book (13) was determined. Our criterion for the presence of dysplasia in lichen planus was Neville reference book (13).

Samples of initial diagnosis which were not confirmed by pathologists or samples without sufficient tissue and inadequate quality or fixation were excluded from the study. Then samples were stained for evaluation of proliferative activity in accordance with the technique of 
AgNOR (argyrophilic nucleolar organizer region) which was characterized by the Jaafari Ashkondi and his colleagues (6) as follows:

Xylenol was used for deparaffinization. Tissue sections were alchemized and then washed in distilled water. Silver staining solution by dissolving a part of volume of $2 \%$ gel in formic acid dissolved in $1 \%$ water and two part of volume of silver nitrate solution dissolved in 50\% water (AgNO3) freshly prepared and embargoed in a dark room. It was allowed to react at room temperature for 20 minutes. The slides were washed with distilled water, and then they were treated in contact with thiosulfate solution $(5 \%)$ for 10 minutes. The slides in varying degrees were washed with acid alcohol and xylene.

Stained slides by two independent pathologists with a light microscope (Olympus ch30 Tokyo, Japon) were evaluated with a magnification of 1000X. AgNOR points were counted in 100 cells of the basal and parabasal layers and were calculated as the mean number of AgNOR per nucleus. Proliferation index (cells with AgNOR dots $\geq 5$ ) was also calculated. The difference in the size of AgNOR was evaluated and groups were defined as follows: Group 1: the same size, group 2: different sizes of dots. Data were analyzed by using statistical software SPSS 20, and using statistical tests Anova, t-test, Chi-square. In this test $\mathrm{P}_{\text {value }}<0.05$ was considered as significant level.

\section{Results}

This study was done on the blocks lichen planus and lichenoid reactions available at the archives of department of Oral \& Maxillofacial Pathology of Babol and Mashhad University of Medical Sciences. Samples were stained by AgNOR technique (argyrophilic nucleolar organizer region) (Figure 1 and 2).

The mean points of AgNOR:
Mean points of AgNOR per core in samples of lichen planus, lichenoid reactions and their subtypes and normal mucosa are summarized in Table 1.

Statistical analysis showed a significant difference in terms of the mean number of AgNOR per core between lichen planus of patients and normal mucosa (P-value $=0.004)$ as well as lichenoid reactions between samples and normal mucosa $(\mathrm{P}$-value $=0.049)$.

The statistical analysis showed statistically significant difference between the mean number of AgNOR per core lichenoid reactions erosive type and reticular lichenoid reactions $(\mathrm{P}$-value $=0.027)$, and lichen planus reticular lichenoid reactions $(\mathrm{P}$-value $=0.043)$ between erosive type lichenoid reactions and normal mucosa ( $\mathrm{P}$ value $=0.004)$, between OLP and normal mucosa (Pvalue $=0.009$ ), between the reticular oral lichen planus and normal mucosa $(\mathrm{P}$-value $=0.007)$ and between oral lichen planus with dysplasia and normal mucosa (Pvalue $=0.019$ ).

\section{Proliferation index:}

Proliferation index of the studied groups is summarized (mean number of cells by more than 5 AgNOR dots per core) in Table 1.

Statistical analysis showed no significant differences between the groups in terms of proliferation index lichen planus, lichenoid reactions, normal mucosa (P-value $=0.073$ ).

The statistical analysis showed that there is a significant difference between groups in terms of proliferation index within the subtypes $(\mathrm{P}$-value $=0.004)$ that indicate this difference was due to the significant difference between the proliferation index erosive lichenoid reaction by reacting Lichenoid reticular (P-value $=0.008$ ) as well as between erosive lichenoid reaction with normal mucosa $(\mathrm{P}$-value $=0.004)$.

\section{The difference in size of AgNOR:}

Table (2) shows qualitative evaluation of AgNOR dots among the studied groups in terms of difference in 
size.Statistical analysis showed no significant difference between the groups in terms of AgNOR dots lichen planus, Lichenoid reactions and normal mucosa $(\mathrm{P}-$ value $=0.245$ ).

Table 1. The mean points AgNOR and proliferation index

\begin{tabular}{|c|c|c|c|c|c|}
\hline \multicolumn{2}{|c|}{ Proliferation index } & \multicolumn{2}{|c|}{ The mean points of AgNOR } & \multicolumn{2}{|c|}{ lesion } \\
\hline \multirow{3}{*}{$7.35 \pm 4.40$} & $8.51 \pm 5.20$ & \multirow{3}{*}{$1.19 \pm 2.13$} & $1.67 \pm 2.19$ & Reticular & \multirow{3}{*}{ lichen planus } \\
\hline & $5.79 \pm 3.73$ & & $0.91 \pm 2.15$ & Erosive & \\
\hline & $7.92 \pm 4.26$ & & $0.78 \pm 2.07$ & $\begin{array}{l}\text { Dysplasia } \\
\text { associated }\end{array}$ & \\
\hline \multirow{2}{*}{$8.13 \pm 4.86$} & $1.59 \pm 0.53$ & \multirow{2}{*}{$0.84 \pm 1.71$} & $0.35 \pm 1.18$ & Reticular & \multirow{2}{*}{$\begin{array}{l}\text { Lichenoid } \\
\text { reactions }\end{array}$} \\
\hline & $9.70 \pm 9.2$ & & $0.86 \pm 2.24$ & Erosive & \\
\hline \multicolumn{2}{|c|}{0} & \multicolumn{2}{|c|}{$0.31 \pm 0.96$} & \multicolumn{2}{|c|}{ Normal } \\
\hline
\end{tabular}

$\underline{\text { Table 2. Classification of study groups in terms of size of AgNOR }}$

\begin{tabular}{lll}
\hline lesion & Group $1^{*}$ & Group 2** \\
\hline Lichen Planus & 11 & 34 \\
Lichenoid reactions & 8 & 22 \\
normal & 7 & 8 \\
\hline
\end{tabular}

*Contain sample of the same size of AgNOR

**Containing samples of different sizes

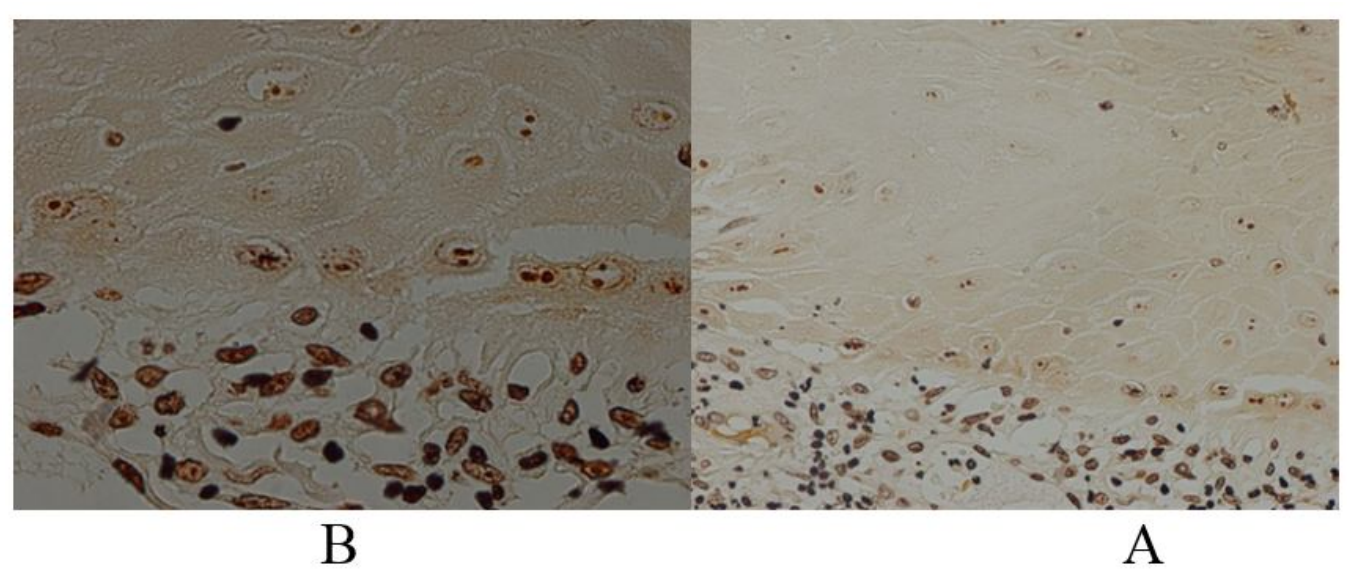

AgNOR stained OLP with dysplasia $($ A $400 \times$ _ B $1000 \times$ ) 


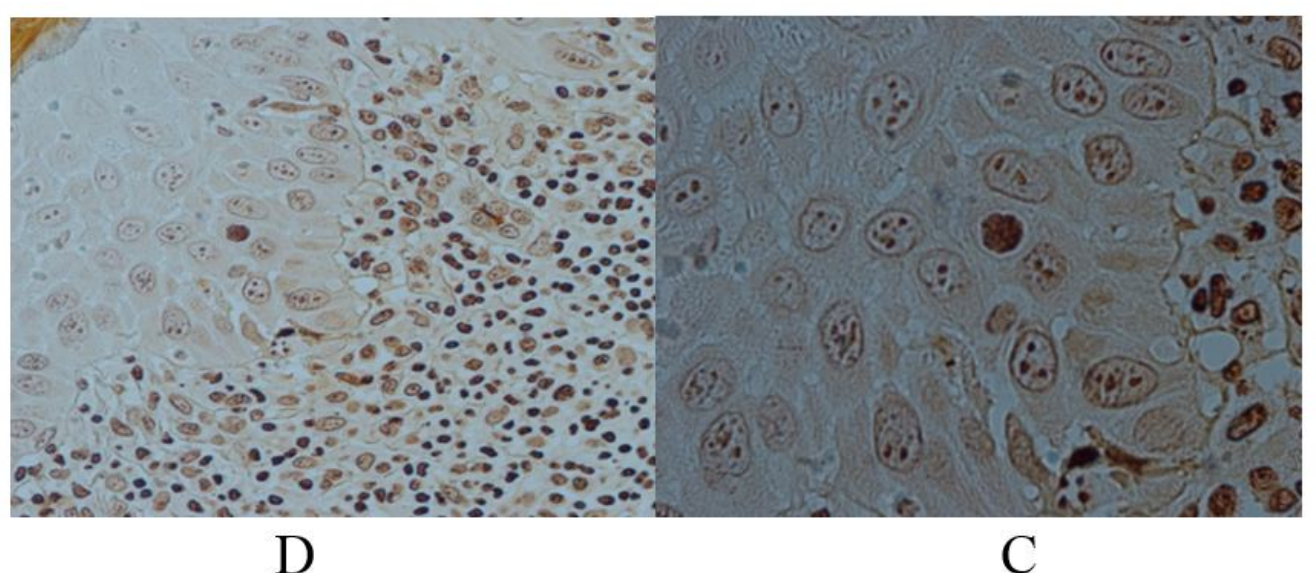

Fig 2. Stained AgNOR erosive lichenoid reactions (a $400 \times$ _ B $1000 \times$ ).

\section{Discussion}

This study aimed to compare lichen planus and lichenoid reactions of cellular proliferative activity by AgNOR method.

However lichenoid reactions are similar to lichen planus, they can cause physical changes (13-14-15) but the approach is very different with regard to two groups of lesions (13). The problem of distinguishing the two lesions on histopathology (13-14-15) is the utilization of proliferation capacity. Our findings show that only differences between subgroups reticular proliferative activity of lesions of lichen planus and lichenoid reactions AgNOR technique is significant. (Pvalue $=0.043$ ).

Acay RR et al. (16) In a similar study, compared the proliferative activity of lichen planus and lichenoid reactions factor Ki67 by immunohistochemistry staining methods and showed that the proliferative activity between the two groups was not significant.

Mega H., et al (14) examined the proliferative activity of lichen planus and lichenoid reactions using immunohistochemically factor Ki67 and PCNA histoplasmosis chemistry and concluded that these results are somewhat consistent with the results of this study. However, in general, proliferative activity lichen planus and lichenoid reactions did not show significant differences in this study, but its reticular kinds showed different proliferative activity from each other which indicates that in the study of Mega $\mathrm{H}$ subgroups of lesions were not investigated.

Acay RR et al. (16) because of high incidence of Ki67 in these lesions and considering factor involved in malignant lesions in the oral cavity due to the increased lesion grading stated that lichen planus and lichenoid reactions have the potential for malignant transformation. This study showed that lichen planus and lichenoid reactions means of AgNOR points are higher than the normal mucosa which shows higher proliferating activity than normal mucosa lesions that means the higher proliferative activity may be is one of the first indicators for malignant transformation. (10) In the study of Karatsaidis A et al (15) about the proliferative activity of reticular and erosive lichenoid oral by using immunohistochemical factor Ki67, they concluded that the Erosive Oral lichenoid has higher proliferative activity of reticular type and normal mucosa similar to the results this study. This increase in activity compared to reticular proliferation in lesion Erosive indicates erosive type is at the disease activity stage. (15)

Increasing proliferative capacity may be one of the first indicators for malignant transformation. (10) Given that lichen planus in some sources considered as a premalignant lesion, it was thought that evaluating the 
capacity of its reproduction through qualitative and quantitative analysis to check AgNOR's possibility of malignant transformation would be helpful.

The study showed that the average of AgNOR at the core of lichen planus such as lichen planus, reticular and erosive are significantly higher than the nucleus of normal mucosa, which reflects the rate of cell proliferation in oral lichen planus. This finding also is consistent with study of Fonseca L et al (17), Majeed AH et al (18), Kulkarni S, et al (19).

Similar to this study, De Carli and colleagues (20) showed a significant difference in terms of average points per core AgNOR were above between reticular lichen planus and normal mucosa that is consistent with our study.

In the studies of Montebugnoli L et al (5), Taniguchi Y et al (21), Hirota $M$, et al (22), on the activity of proliferative lichen planus patients mouth using factor Ki67, showed proliferative active was significantly higher than normal samples.

According to the studies of Taniguchi Y et al (21) and Acay RR et al. (16) the increasing cellular proliferation in Lichen planus is a secondary phenomenon due to damage of keratinocytes by infiltration of mononuclear cells of mucosa.

Lichen planus may indicate higher proliferation rates of these lesions are likely malignant transformation. (10) Although in some papers (kulkarni and colleagues (19) argue that the AgNOR only reflects the activity of cell proliferation and does not show malignant transformation.

However, in the study of De Susa F, et al (10) AgNOR values reported in oral lichen planus lesions is significantly less epithelial dysplasia and squamous cell carcinoma which implies fast proliferation of smaller and more relaxed policy for oral lichen planus compared to epithelial dysplasia and squamous cell carcinoma.

As well as our study, the mean number of AgNOR per core, there was no difference in size of the AgNOR, proliferation index of dysplastic and non-dysplastic (including reticular and erosive).

Due to the lack of significant differences between dysplastic and non-dysplastic OLP values of AgNOR in cases of dysplastic and non-dysplastic cell proliferation rate indicates that the AgNOR technique cannot be used to detect the presence of dysplasia in the oral lichen planus patients. This finding is consistent with the findings of Jaafari Ashkoandi et al (6).

With regard to proliferative index (the number of cells with $\mathrm{AgNOR} \geq 5$ ) significant difference between normal epithelium and lichen planus lesions were observed. In this sense the study Majeed AH et al. (18) is in contrast of our study which could be attributed to two different cut off point for proliferation index. Also, qualitative analysis of AgNOR points showed no significant differences between the three groups (OLP, lichenoid reactions, and normal mucosa) which is inconsistent with study of Kulkarni et al (19).However, this contrast may be referred to the parameters of qualitative evaluation of AgNOR dot.

In the investigation of proliferative activity of reticular and erosive oral lichen planus of this study there were no significant differences between the two groups that is consistent with study of Montebugnoli and colleagues (5) and YTaniguchi and colleagues (21) Majeed AH et al. (18). This means that the Sub-proliferative activities of lichen planus (reticular and erosive) are somewhat similar to others and there was not significant difference in the risk of malignant transformation of erosive and reticular.

The study of Pramod Redder C et al (23) indicates OLP had high proliferation activity compared to reticular and plaque using immunohistochemically PCNA. Their findings are inconsistent with this study, even though we used the proliferative activity of AgNOR techniques in our evaluation. Due to the difference between this study and the study of Pramod Redder $\mathrm{C}$ et al it can be said that more studies are needed for closer inspection. 


\section{Conclusion}

Since the number of AgNOR in oral lichen planus reticular significantly was higher than reticular lichenoid reactions, we can use AgNOR techniques to differentiate challenging types of lichen planus reticular from lichenoid reactions reticular in histopathologic specimens.

The lack of significant difference between dysplastic and non-dysplastic OLP in AgNOR values indicates that the AgNOR technique cannot be used to detect for the presence of dysplasia in the oral lichen planus patients. Due to the higher proliferative activity than normal mucosa lichen planus and lichenoid reactions may have the possibility of malignant transformation in these lesions. So successive patient's follow-up for early diagnosis of potential malignancy can be helpful.

\section{References:}

1. van der Waal I. Oral Lichen Planus: Diagnosis and Management. J Dent Indonesia 2015;22(3):89-92.

2. Do Prado RF, Marocchio LS, Felipini RC. Oral lichen planus versus oral lichenoid reaction: Difficulties in the diagnosis. Indian J Dent Res 2009; 20(3):361-4.

3. Glick M. Burket's Oral Medicine, 12th ed .Shelton: PMPHUSA; 2015. P:104.

4. Sagari SH, Sanadhya S, Doddamani M, Rajput R. Molecular markers in oral lichen planus. $\mathrm{J}$ oral maxillofac pathol 2016; 20(1): 115-21.

5. Montebugnoli L, Farnedi A, Marchetti C, Magrini E, Pession A, Foschini MP. High proliferation activity and chromosomal instability in oral lichen planus. Int J. Oral Maxillofac Surg 2006; 35:1140-4.

6. Jaafari-Ashkvandi Z, Fatemi FS, Evaluation of Proliferation Activity in Dysplastic and Nondysplastic Oral Lichen planus Through the Analysis of Argyrophilic Nucleolar Organizer Regions. J Craniofac Surg.2013; 24(3):788-91.

7. Epstein JB, Wan LS, Gorsky M, Zhang L. Oral lichen planus: progress in understanding its malignant potential and the implications for clinical management. Oral Surg
Oral Med Oral Pathol Oral Radiol Endod 2003; 96(1):327.

8. Laeijendecker R, Van Joost T, Kuizinga MC, Tank B, Neumann HA. Premalignant nature of oral lichen planus. Acta Derm Venereol 2005; 85(6):516-20.

9. Gonzalez-Moles MA, Scully C, Gil-Montoya JA. Oral lichen planus: controversies surrounding malignant transformation. Oral Dis 2008; 14(3):229-43.

10. De Sousa F, Paradella TC, Carvalho YR, Rosa L. Comparative analysis of cell proliferation ratio in oral lichen planus, epithelial dysplasia and oral squamous cell carcinoma. Med Oral Patol Oral Cir Bucal 2009; 14 (11):e563-7.

11. Tomazelli KB, Modolo F, Rivero ERC. Evaluation of AgNORs in Oral Potentially Malignant Lesions. J Oncol 2015;2015:218280.

12. Elangovan T, Mani M, Malathi N. Argyrophilic nucleolar organizer regions in inflammatory, premalignant,and malignant oral lesions: a quantitative a qualitative assessment. Indian J Dent Res 2008; 19(2):141-6.

13. Neville B, Damm D, Allen C, Bouquot J. Oral and maxillofacial patholpgy.4th ed. ST.Louis: Sunders Elsevir; 2016. P:729.

14. Mega H, Jiang WW, Takagi M. Immunohistochemical study of oral lichen planus associated with hepatitis C virus infection, oral lichenoid contact sensitivity reaction and idiopathic oral lichen planus. Oral Dis 2001;7(5):296305.

15. Karatsaidis A, Schreurs O, Helgeland K, Axéll T, Schenck K. Erythematous and reticular forms of oral lichen planus and oral lichenoid reactions differ in pathological features related to disease activity. J Oral Pathol Med 2003;32(5):275-81.

16. Acay RR, Felizzola CR, De AraÚjo NS, De sousa SOM.Evaluation of proliferative potential in oral lichen planus and oral lichenoid lesions using immunohistochemical expression of p53 and Ki67. Oral Oncol 2006; 42:475-80. 
17. Fonseca L, Do Carmo M. Identification of the AgNORs, PCNA and ck16 proteins in oral lichen planus lesions. Oral Dis 2001; 7(6):344-8.

18. Majeed AH. The Diagnostic Value of Nucleolar Organizer Region in Oral Lichen Planus (a preliminary study). J College Dentistry 2004;16(1):35.

19. Kulkarni S, Mody R, Jindal S, Sohi RS, Kaur B. Silver binding nucleolar organizer regions in oral submucous fibrosis, lichen planus, leukoplakia and squamous cell carcinoma. J Can Res Expi Oncol 2009;1(12):15-9.

20.De Carli JP, Da Silva SO, Sandini Linden MS, Busin CS, Paranhos LR, Couto Souza PH. Evaluation of Cellular Proliferation Activity in Patient with Oral Lichen Planus and Hepatitis C through AgNOR Method. Braz Dent J 2014; 25(6):461-5.
21. Taniguchi $Y$, Nagao $T$, Maeda $H$, Kameyama $Y$, Warnakulasuriya KAAS. Epithelial cell proliferation in oral lichen planus. Blackwell Science Ltd 2002; 35(suppl.1):103-9.

22. Hirota M, Lto T, Okudela K, Kawabe R, Yazawa T, Hayashi $\mathrm{H}$, et al .Cell proliferation activity and the expression of cell cycle regulatory proteins in oral lichen planus. J Oral Pathol Med 2002; 31: 204-12.

23. Pramod Redder C, Pandit S, Desai D, Suresh Kandagal V, Ing.aleshwar PS, Shetty SJ, et al. Comparative analysis of cell proliferation ratio in plaque and erosive oral lichen planus: An immunohistochemical study.Dent Res J (Isfahan) 2016; 11(3):316-20. 